\title{
Statistical Analysis of Some Gas Chromatography Measurements
}

\author{
Karen Kafadar* and Keith R. Eberhardt*
}

National Bureau of Standards, Washington, DC 20234

November 3, 1982

\begin{abstract}
The National Bureau of Standards has certified Standard Reference Materials (SRMs) for the concentration of polychlorinated biphenyls (PCBs) in hydrocarbon matrices (transformer and motor oils). The certification of these SRMs involved measurements of extremely small concentrations of PCBs made by gas chromatography. Despite the high accuracy of the measurement technique, the correlated data cannot be analyzed in a routine independent manner. A linear model for the measurements is described; its complexity encourages the use of simpler exploratory methods which reveal unexpected features and point the way towards obtaining valid statistical summaries of the data.
\end{abstract}

Key words: exploratory analysis; linear models; median polish; robust estimates; statistical methods; uncertainty statement.

\section{Introduction}

Exploratory methods in data analysis are used in many fields of application. These methods are typically used on messy data because they are robust in nature; that is, they are insensitive to unexpected departures from an assumed model (e.g. outliers, non-normality). However, they can also provide valuable insight even for so-called "clean" data. This paper discusses an example of a measurement process for which an exploratory approach can reveal particularly interesting or unexpected trends even in extremely precise measurements.

This paper is about the application of some simple exploratory techniques discussed by Tukey [1] ${ }^{1}$ and others to several sets of measurements obtained by gas chromatography. These data were taken at the National Bureau of Standards between November 1981 and March 1982 and are described in section 2. Despite the high accuracy of the method, the correlated measurements cannot be treated in a routine independent manner. A linear model for the data is proposed in section 3 , and a robust analysis of this model, based on exploratory methods, is described in section 4. Conclusions are summarized in section 5 .

\footnotetext{
*Statistical Engineering Division, National Engineering Laboratory.

${ }^{1}$ Figures in brackets indicate-the literature references at the end of this paper.
}

\section{The Data}

\subsection{Description of the SRM for PCBs in Oils}

The National Bureau of Standards has certified Standard Reference Materials (SRMs) for the concentration of polychlorinated biphenyls (PCBs) in hydrocarbon matrices, namely, transformer and motor oils. PCBs are toxic contaminants; their chemical and thermal stability makes them commercially useful but also leads to their persistence in the environment. PCBs are formulated as liquid mixtures of congeners and were manufactured in the United States under the trade name Aroclor. These mixtures have been used extensively in this country as coolants in high-voltage electrical components and may be introduced into the environment when these components are serviced, repaired, or discarded. Since the PCB fluid physically resembles lubricating oil, there have been instances in which PCBs have been added mistakenly to motor oils being collected for recycling purposes.

For these reasons, it is important to be able to measure small concentrations of PCBs. These measurements are now aided by NBS Standard Reference Material 1581 which provides certified concentrations of two congeneric PCB mixtures in two forms, known as Aroclor 1260 and Aroclor 1242. Four materials constitute this SRM, identified as Aroclor 1260 in motor oil, Aroclor 1260 in transformer oil, Aroclor 1242 in motor oil, and Aroclor 
1242 in transformer oil. Large lots of these materials were prepared, carefully blended and dispensed into 5-mL amber ampoules, yielding about 4000 ampoules of each material. Six sample vials of each material were selected at random for chemical analysis.

This paper describes the statistical analysis of the measurements on the selected sample vials. The method of analysis was the same for all four types of material; hence, we will limit our discussion to its application on Aroclor 1260 in motor oil, for the most part.

\subsection{Measurement Techniques}

A method for chemical analysis of PCBs in oil was developed at the National Bureau of Standards and employs both liquid and gas chromatography for analysis. Quantitation is performed by a calibrated internal standard (IS) technique which is described in detail by Chesler et al. [2]. Basically, this technique works in the following manner. First, a sample containing a known amount of analyte (in this case, PCB) and known amounts of one or more internal standards is analyzed. The instrumental responses, measured as individual peak areas, are recorded, and the relative responses between the analyte and the internal standards (ISs) are ascertained. Second, known amounts of the internal standards are added to a sample for which the concentration of the analyte is desired, and the sample is subsequently analyzed. Then, using the relative response factors previously ascertained, the concentration of the analyte is determined from the ratio of the responses between the analyte and internal standard. In the particular experiments discussed below, each measurement of the analyte gave 10 separate measurable responses (i.e. 10 distinct peaks) and each of the three internal standards gave one measurable response. Figure 1 shows a sample chromatogram from one of the analyses. Thus, since analyte concentration can be calculated from any combination of one internal standard plus one analyte peak, the analyte concentration can be calculated in many ways for each single analysis.

\subsection{A Formula for Determining Aroclor Concentration}

The response factors, i.e., the ratios (peak area)/(concentration), are not necessarily constant from run to run. In fact, the response factor for a given peak is subject to many kinds of instrumental variation, such as the electronic detector and heat source differences inside the instrument and variations in the flow rate of material through the gas chromatographic column. But, to a large extent, these variations affect all peaks (including those that arise from the internal standard) in the same way.
Therefore, on a given "oceasion" when several runs are all reasonably close together in time, the ratio

\section{(peak area for Aroclor)/(concentration of Aroclor)}

(peak area for IS)/(concentration of IS)

should be constant for each run and each solution, whether it is a sample solution or a specially-prepared calibration solution. In principle, then, (1) should be the same for the sample and the calibration material; i.e.,

$$
\frac{P(I, s) / C(I, s)}{P(a, s) / C(a, s)}=\frac{P(I, c) / C(I, c)}{P(a, c) / C(a, c)}
$$

where

$P$ denotes peak area

$C$ denotes concentration

$I$ indexes internal standard peak

a indexes Aroclor peak

$s$ denotes sample solution

c denotes calibration solution.

Therefore, a calibration solution is passed through the chromatograph at approximately the same time a sample solution is run, and the coneentration of the Aroclor in a sample, based on a given peak area, may be determined as

$$
C(a, s)=\frac{P(I, c)}{P(a, c)} \times \frac{P(a, s)}{P(I, s)} \times \frac{C(I, s)}{C(I, c)} \times C(a, c) .
$$

Note that different peaks will yield different values for concentration.

The concentration of a substance in a solution is very nearly the mass of the analyte divided by the mass of the oil in that solution. In the actual experiment, the weight of the internal standard was measured and held constant for both the ealibration solution and the sample solution. So in this experiment,

$$
\frac{C(I, s)}{C(I, c)}=\frac{W(I, s) / W(\text { oil }, s)}{W(I, c) / W(\text { oil }, c)}=\frac{W(\text { oil }, c)}{W(o i l, s)}
$$

where $\mathrm{W}$ denotes weight. Therefore, the formula

$$
C(a, s)=\frac{P(I, c)}{P(I, s)} \times \frac{P(a, s)}{P(a, c)} \times \frac{W(o i l, c)}{W(o i l, s)} \times C(a, c),
$$

is actually sufficient for determining Aroclor concentration. 


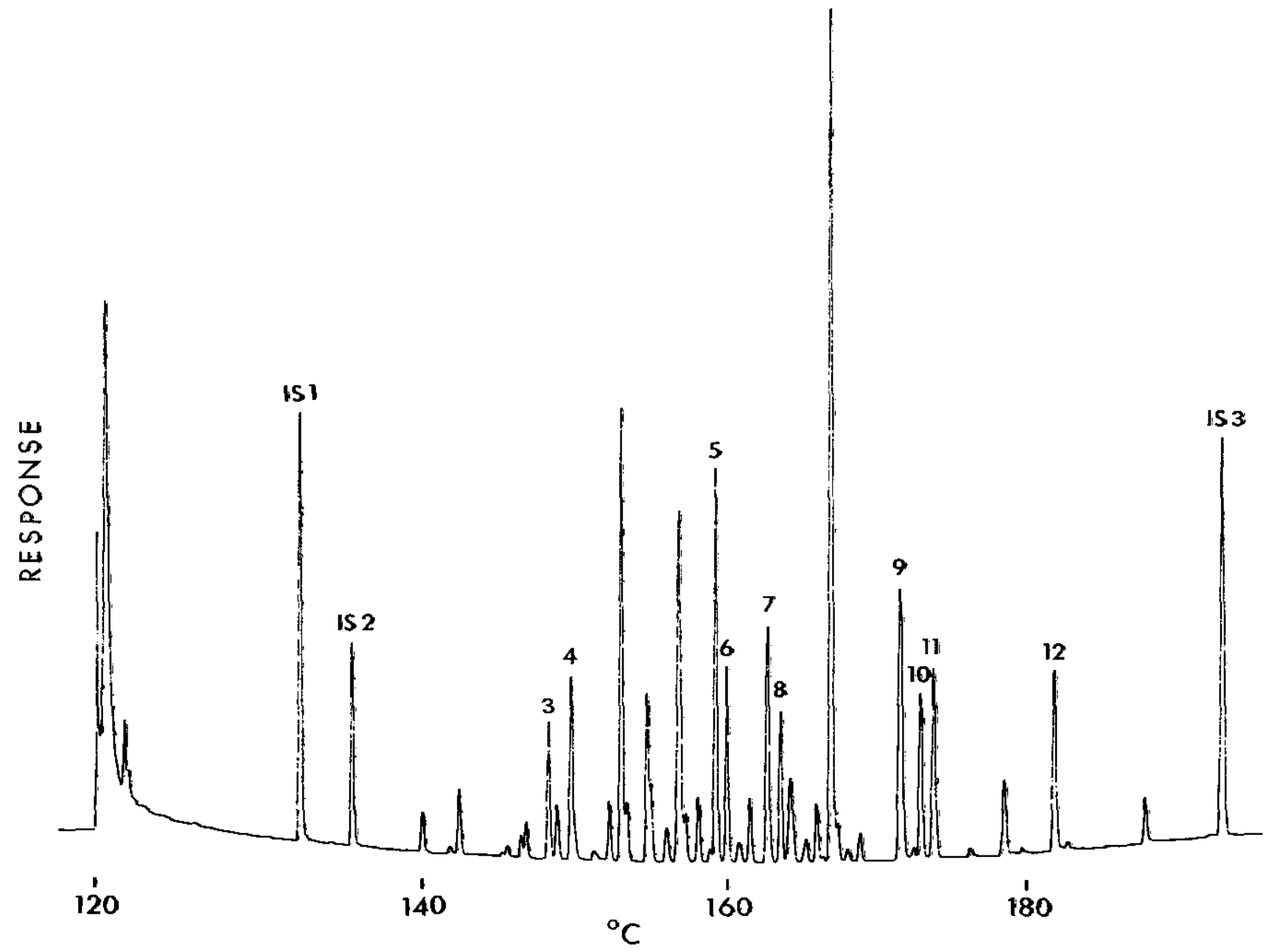

FIGURE 1. A gas chromatogram from one subsample of Aroclor 1260 in motor oil. IS 1, IS 2, IS 3 denote peaks from three internal standards; 3 through 12 denote Aroclor peaks. The horizontal scale indicates the temperature levels in the gas chromatographic column at which the compounds are eluted.

\subsection{Experiment Design}

For each material, three subsamples were analyzed from each of the six selected ampoules. On a given occasion (2-3 hour period), two subsamples, one from each of two ampoules, were run along with one calibration solution. Display 1 presents the experimental design. Given the method of preparation, a high degree of homogeneity among the ampoules was expected. With these limited data, tests on the variance among the six ampoules showed no evidence of inhomogeneity compared to the overall variability of the measurements.

As a result of the design in display 1 , the calculated values of concentration for two subsamples analyzed on the same occasion are not independent. In eq (2), these two calculated concentrations would share the same value of the ratio $P(I, c) / P(a, c)$. Thus, for a given (internal standard peak, Aroclor peak) pair, the 18 concentration values obtained from six ampoules each with three replications are not independent. Furthermone, since there are many pairs of internal standards and Aroclor peaks for each sample, this design leads to a large number of calculated concentration values having strong and complex interdependencies. The challenge is to take proper account of these dependencies in obtaining an overall certified value and uncertainty statement for the SRM certificate.

\section{A Linear Model for the Measurements}

The basic properties of the gas chromatography procedure described by eq (1), plus the rules and provisos mentioned in the surrounding text, can be represented by a linear statistical model. Denoting $\log _{10}$ (peak area) by $Y$, these considerations lead to the model in display 2 . This model is an unbalanced partially nested and partially crossed analysis of variance model with a total of 211 
Display 1-Experimental design

Allocation of Subsamples and Calibrations to Oceasions on Which GC Analysis Runs Were Made*

\begin{tabular}{|c|c|c|}
\hline Occasion & Sample ID & Calibration Solution \\
\hline 1 & $S 1, S 2$ & $\mathbf{A}$ \\
\hline 2 & S1, S2 & A \\
\hline 3 & $\mathrm{S1}, \mathrm{S2}$ & $\mathbf{A}$ \\
\hline 4 & S3, $\$ 4$ & $\mathrm{~B}$ \\
\hline 5 & S3, S4 & B \\
\hline 6 & $\mathrm{S3}, \mathbf{S 4}$ & B \\
\hline 7 & $55, \$ 6$ & C \\
\hline 8 & S5, S6 & C \\
\hline 9 & 55,56 & C \\
\hline
\end{tabular}

*Note: The order in which the two samples and one calibration were run witbin each occasion was varied.

Display $2-$ Linear model for $Y=\log _{1 \text { (peak area })}$

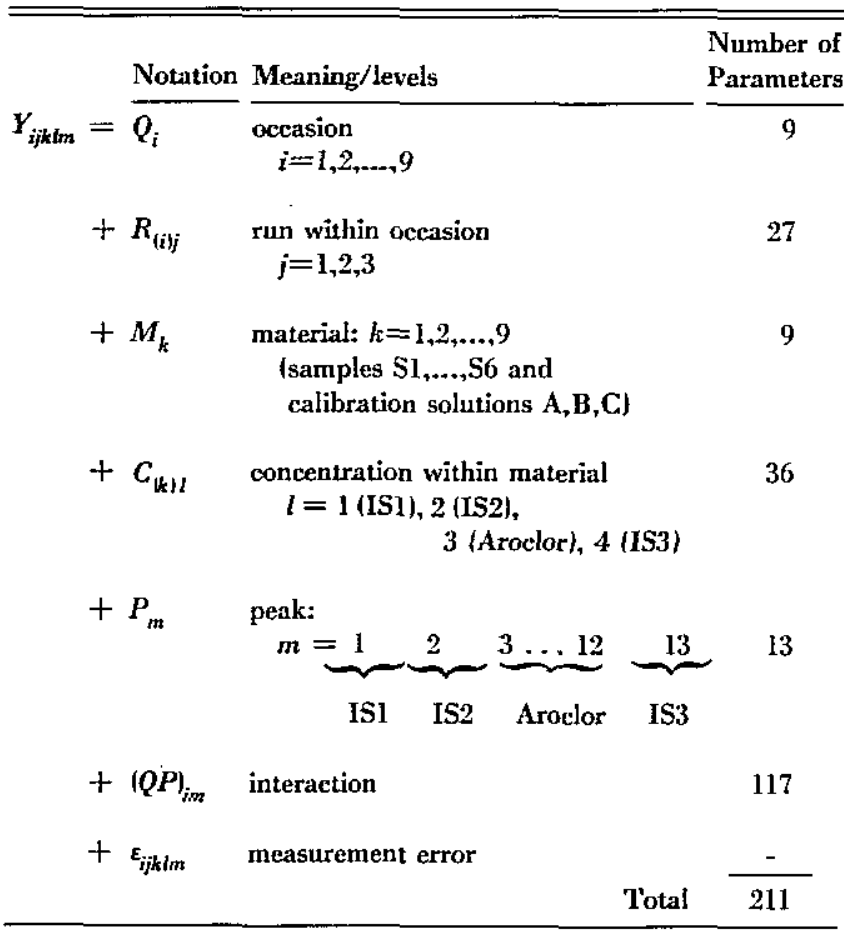

parameters and 351 observations. The 351 measured peak areas come from 9 occasions with 3 runs each (one calibration and two SRM subsamples-see display 1), for a total of 27 runs, times 13 peaks per run.

While we feel that this linear model provides a reasonably complete and realistic representation of the measurements, there were several reasons which prevented its use as a sufficient guide for the data analysis.

First, some implicit constraints in the model are difficult to incorporate in an analysis with available statistical computer software. These constraints arise from the fact that Aroclor concentrations in the calibration solutions are aetually known before any peak areas are measured. Similarly, the ratios of concentrations for the internal standards are controlled, as deseribed in section 2.3 above. Taken together, these considerations imply that there are actually only six free parameters (corresponding to the concentrations of Aroclor in the six selected ampoules) among the 36 model terms denoted $C_{(k) !}$. In contrast, the statistical computer packages which can handle a problem of this size and of incomplete rank do not have any provision for imposing the necessary constraints, so that the $C_{(k) 1}$ terms in the model are treated (inappropriately) as 36 free parameters. A future manuscript will give a detailed look at this and other aspects of the least squares treatment of these data under the linear model.

Another reason for not using the model directly is the probable lack of homogeneity in the variances of the logarithms of the measured peak areas, which is implicitly assumed in computer packages. Evidence of variance inhomogeneity arose from the concerns of the chemists and was exhibited in the data (see section 4.3).

Although a least squares analysis of the model was not used to obtain the certified value and uncertainty for the SRM certificate, it is instructive to compare the data summaries suggested by least squares with the more robust alternative actually used. For example, for each replication on each sample, it is possible to calculate the Aroclor concentration in 30 different ways by using one of the three internal standards with any one of the 10 available Aroclor peaks. It can be shown that a least squares fit of the model implies that one should summarize those 30 possible concentration values by taking their arithmetic mean value (in log scale). In the following sections, some useful alternatives to simply averaging $\log ($ concentration) values will be described.

\section{An Exploratory Analysis}

\subsection{Concentrations Revisited}

As illustrated in display 1 , samples S1 and S2 were run on the same occasion as a calibration solution denoted by A; likewise, samples S3 and S4 were run with calibration $B$, and samples S5 and S6 were run with calibration C. Three replications were performed on each occasion. Transforming eq $\left(2^{\prime}\right)$ via logarithms, a calculation of $\log$ (concentration) may be described as

$$
\begin{aligned}
\mathrm{Z} & =\log (\underbrace{\text { weight of oil } \times \text { one IS peak/one Aroclor peak })}_{\text {calibration }} \\
& -\log (\underbrace{\text { weight of oil } \times \text { same IS peak/same Aroclor peak })(3)}_{\text {sample }} \\
& +\log (\text { concentration of Aroclor in calibration solution). }
\end{aligned}
$$


Notice that there are (3 calibrations) $\times(3$ replications) $x$ (3 IS peaks) $\times(10$ Aroclor peaks $)=270$ values for the first term in eq (3), and (6 samples) $\times 3 \times 3 \times 10=540$ values for the second term. The concentration in each calibration solution is determined gravimetrically once for all three replications, so there are only three values for the third term in (3) (typically, about $\log _{10}(100.01$ $\mu \mathrm{g} / \mathrm{g}) \approx 2$ ).

\subsection{Summarizing the Doto}

The quantity $U=\log _{10}$ ( weight of oil $\times$ (IS peak) /(Aroclor peak)], for either the calibration or the sample, is of interest, for its value can be determined entirely from the measurements made during a single run. Corresponding values of $U$ must be combined for the appropriate sample and calibration within a single occasion. If we consider the measurements based on a particular internal standard and peak area, we can reduce the data for two samples by averaging the $U$-values from the independent replications of two samples, and combining this average with the value from the calibration solution on the same occasion. Thus, for each peak and internal standard, we can obtain nine summary values, one for each replication on each of three calibration solutions. Let us denote these "summary values" by $Z_{x j i n}$, where $\alpha=\mathrm{A}, \mathrm{B}, \mathrm{C}$ identifies the ealibration solution, $j=$ $1,2,3$ indexes replications, $l=1,2,3$ indexes the internal standard, and $n=3,4, \ldots, 12$ indexes the Aroclor peak. Thus, $\boldsymbol{Z}_{a j \mathrm{j}, \pi}$ is the average $\log$ (Aroclor concentration) for the two samples which were nun on the $j^{\text {th }}$ replication with calibration $\alpha$, using internal standard $l$ and Aroclor peak $\pi$. The values $Z_{x y / n}$ are displayed schematically in figure 2 .

Figure 2 suggests that further summarization is possible across those values that are estimating the same quantity. We can do this in two stages, corresponding to the two two-way layouts that are shown. If we analyze the data via the least-squares model of section 3 , the order of summarization will not matter, and we can simply average all $9 \times 30=270$ values together. However, these values are not independent, and obtaining a standard error for such a grand average is not simple.

Clearly, the 30 values in the secondary layout (b) of figure 2 are highly eorrelated. However, a "typical value" from the 30 values $Z_{a j t n}, l=1,2,3, n=3,4, \ldots, 12$, will be strictly independent of any $Z_{\alpha^{\prime} j_{n}}\left(\alpha \neq \alpha^{\prime}, j \neq j^{\prime}\right)$. It will simplify our uncertainty assessment from this four-way table if we reduce each secondary table containing the correlated values to a single "typical value" representing these numbers. Having fourd such a "typical value," it may be placed in the appropriate cell of (a) of figure 2, where we are assured that the resulting nine values will be independent. A one-way analysis of variance

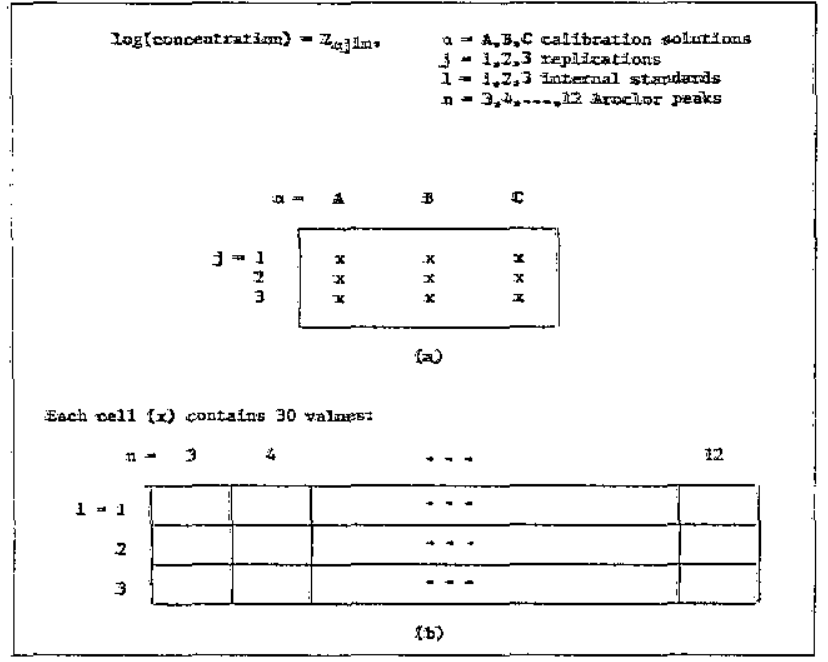

Figdre 2. Schematic layout for summary values of $\log _{10}$ (concentration).

on these nine values may then be performed, and an appropriate standard error obtained herein.

Thus, the analysis proceeds in two stages in which we:

1) Obtain a "typical value" from the entries $Z_{\mathrm{\alpha} i k s}$ for each $\alpha=A, B, C$ and $j=1,2,3$ (replications), and,

2) Analyze the nine "typical values" $Z_{\alpha j}$.. by a standard one-way analysis of variance.

Since chemical reasoning leads us to expect the data in some eells of the secondary tables to be much less reliable than those in other cells, we chose to use two-way median polish to obtain a summary value for the table. This procedure is analogous to a two-way analysis of variance, but is insensitive to large deviations from the typical value which might occur in the data. (An example illustrating the procedure is given in the Appendix.) Furthermore, the correlation among the 30 values in the table will not worry us at this stage since we are interested only in reducing the table to a single typical value.

\subsection{Summarizing the Dependent Pieces: Median Polish}

It is interesting to compare the summary value obtained from median polish with the corresponding summary (the grand mean) obtained from a two-way analysis of variance of the same data.

The differences between the median polish typical value and the grand mean of the 30 values, for each of the nine two-way tables, are illustrated in figure 3 . Notice that the median polish typical value differs from the grand mean whenever one group of values (corresponding to a given internal standard) does not agree with the other two. The median polish typical value gravitates towards the heaviest concentration of values, downplaying the influence of a 
group which is some distance away (e.g., internal standard 3 in the third replication using calibration A). In contrast, the grand mean gives all vahues equal weight, regardless of their relative positions among the others. The grand mean may not be appropriate, given the possibility of chemical reasons for all values from a given internal standard to be high or low. Notice also from figure 3 that the data exhibit evidence of variance inhomogeneity, as. mentioned in section 3. For these reasens, we adopt the median polish typical values as the summary values for this problem.

\subsection{Summorizing the Independent Pieces: One-way Analysis of Variance}

The nime median polish typieal values for Aroclor 1260 in motor oil are shown in the top portion of display 3 .
Since different replications involved preparation of distinct subsamples of material, these nine entries are independent. Furthermore, they are already summaries of $3: 0$ values from peak $\times$ IS combinations. By virtue of central limit theorem considerations, we feel safe in analyzing this table using a standard one-way anakysis of variance model with normal errors.

It is possible that differences among the groups identified by ealibration solutions condd be introduced into the data at the stage when the chemists addd the internal standard "spikes"' to the sample, or when the calibration solutions are prepared. Since the results of these operations are treated as values known without error, errors introduced at this stage would persist as systematic errors between the calibration solution groups. Flowever, the F-tests. for these group effeets were all clearly nonsignificant, with computed $\alpha$-levels ranging from 35

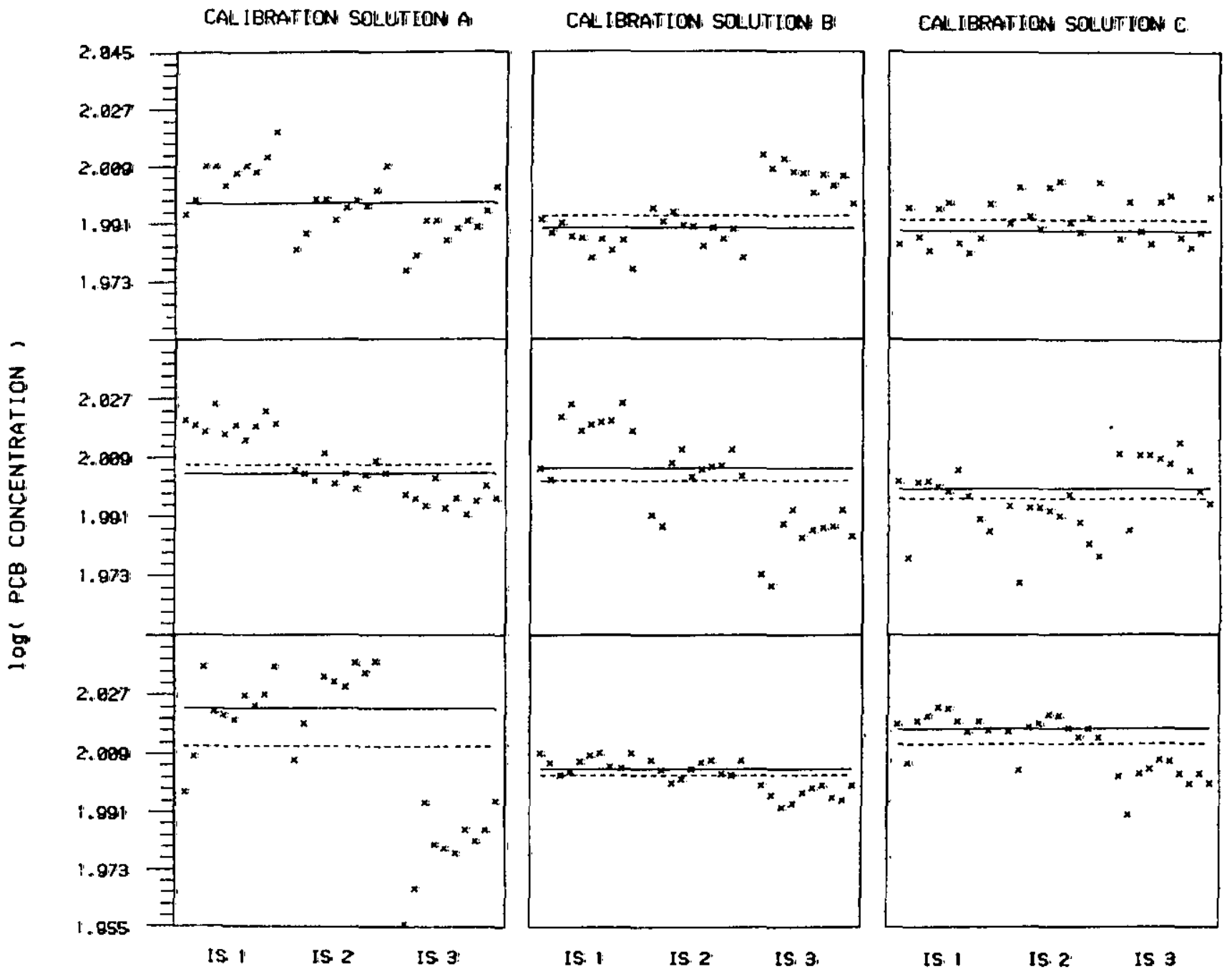

FIGURE 3. Median polish typical values (solid) and grand means (dot) of 30, values of log(concentration): Each set of 10 points refers to a different internal standard. The leftmost graphs are from the replieations using calibration A; the middle are from those using calibration B; and the rightmost are from those using calibration $\mathbb{C}$. 
Display 3-One-way analysis of variance for nine "typical values" of log(concentration) for Aroclor 1260 (motor)

Calibration Solution:

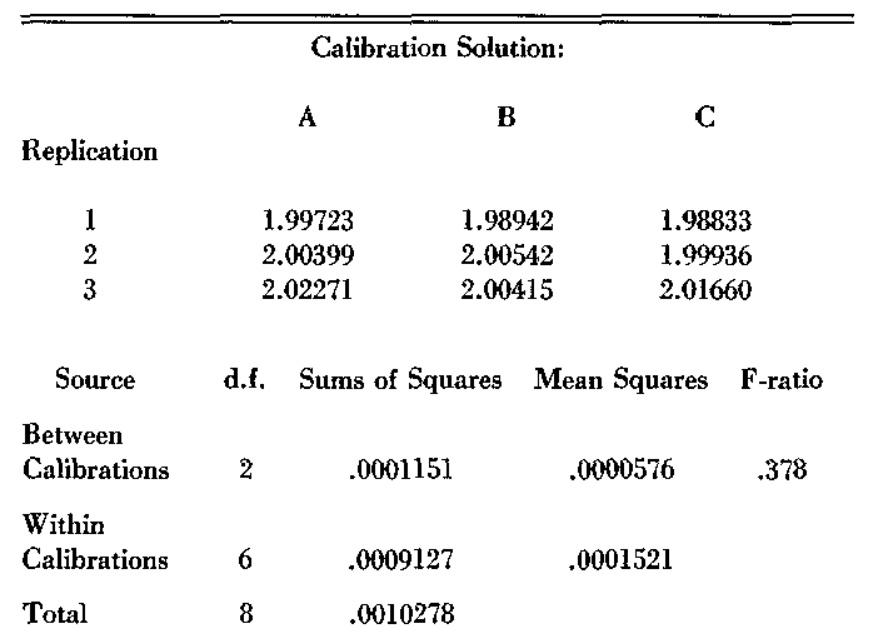

Student's t confidence interval for grand mean (8 d.f.): (1.99431, 2.01174)

percent to 87 percent for the four materials studied. The one-way analysis of variance for Aroclor 1260 (motor) is shown in display 3 .

In the absence of between-group errors, it is reasonable to treat the nine values in our one-way table as independent and identically distributed observations on the Aroclor concentration. Taking this approach, the standard error of the mean of the nine values is estimated in the usual way as $s / \sqrt{9}$. The results of these calculations for all four materials are summarized in table 1.

TABLE 1. Summary Values for Concentrations of PCBs in Oils

\begin{tabular}{|c|c|c|c|c|}
\hline & \multicolumn{2}{|c|}{$\begin{array}{c}\text { Aroclor } 1260 \\
\text { (3 internal standards) }\end{array}$} & \multicolumn{2}{|c|}{$\begin{array}{c}\text { Aroclor } 1242 \\
\text { (2 internal standards) }\end{array}$} \\
\hline & $\begin{array}{l}\text { Motor } \\
\text { Oil }\end{array}$ & $\begin{array}{c}\text { Transformer } \\
\text { Oil }\end{array}$ & $\begin{array}{l}\text { Motor } \\
\text { Oil }\end{array}$ & $\begin{array}{c}\text { Transformer } \\
\text { Oil }\end{array}$ \\
\hline $\begin{array}{l}\log (\text { Concentration), } \\
\text { mean of typical } \\
\text { values from median } \\
\text { polish }\end{array}$ & 2.0030 & 2.00027 & 2.003 & 2.0017 \\
\hline $\begin{array}{l}\text { Standard error for } \\
\text { log(concentration) }\end{array}$ & .0038 & .0050 & .0027 & .0023 \\
\hline $\begin{array}{l}95 \% \text { Confidence } \\
\text { limits (log scale) }\end{array}$ & $\begin{array}{r}(1.9943, \\
2.0117)\end{array}$ & $\begin{array}{r}(1.9912, \\
2.0142)\end{array}$ & $\begin{array}{r}(1.9941, \\
2.0064)\end{array}$ & $\begin{array}{l}(1.9964, \\
2.0070)\end{array}$ \\
\hline Concentration & 100.69 & 100.62 & 100.07 & 100.39 \\
\hline $\begin{array}{l}95 \% \text { Confidence } \\
\text { limits (original } \\
\text { scale): }(\mu \mathrm{g} / \mathrm{g})\end{array}$ & $\begin{array}{l}\text { (98.70, } \\
102.74)\end{array}$ & $\begin{array}{l}(97.99 \\
103.32\}\end{array}$ & $\begin{array}{l}\{98.65, \\
101.48\}\end{array}$ & $\begin{array}{l}(99.18, \\
101.62)\end{array}$ \\
\hline
\end{tabular}

\subsection{Peak Effects}

As in a classical analysis of variance, median polish yields estimates of the effects from the data. It is instructive to examine these estimates, particularly in cases presenting anomalies among them. Nine median polishes were performed to obtain the entries for the layout in (a) of figure 2. The resulting peak effects are plotted in figure 4. Notice that in all cases the median peak effect is zero (by the nature of the median polish analyses) and that in several cases peaks 3 and 4 (first and second from the left) are substantially lower than the others. The average across all nine independent replications is plotted in figure 5, shown with limits of one standard error. Graphs such as this one suggest looking for chemical explanations for the low values on peaks 3 and 4 .

\section{Conclusions}

Four data sets consisting of gas chromatography measurements were analyzed for purposes of SRM certification and for eliciting unusual features in the data. A linear model was proposed, but practical difficulties limit its usefulness for the problem at hand. Exploratory techniques led not only to a certified value and uncertainty but also to the investigation of chemical causes for unusual patterns in the effects.

The proposed analysis for these data is not specific to an experiment of this form-i.e., GC measurements using multiple internal standards and multiple Aroclor peaks, with one calibration solution for every two sample solutions. Rather, it illustrates how the data from independent runs yielding several, but dependent, answers, can be analyzed without resorting to complicated linear models that can sometimes be unwieldly. Basically, our general approach proceeds in two stages:

1) The structure for the dependent pieces is determined, and the pieces are reduced to an appropriate summary value (e.g., median, median polish typical value, or another location estimator), and

2) The structure for the independent pieces is identified, and the data analyzed accordingly.

Since the data at the second stage are independent, standard techniques for obtaining uncertainty statements apply.

As a summarizing technique for the first stage, we used median polish to provide a robust summary for each data set, leading to the values reported in table 1 . Without exploratory analysis, classical techniques may well have obscured some of the interesting features in the data. 


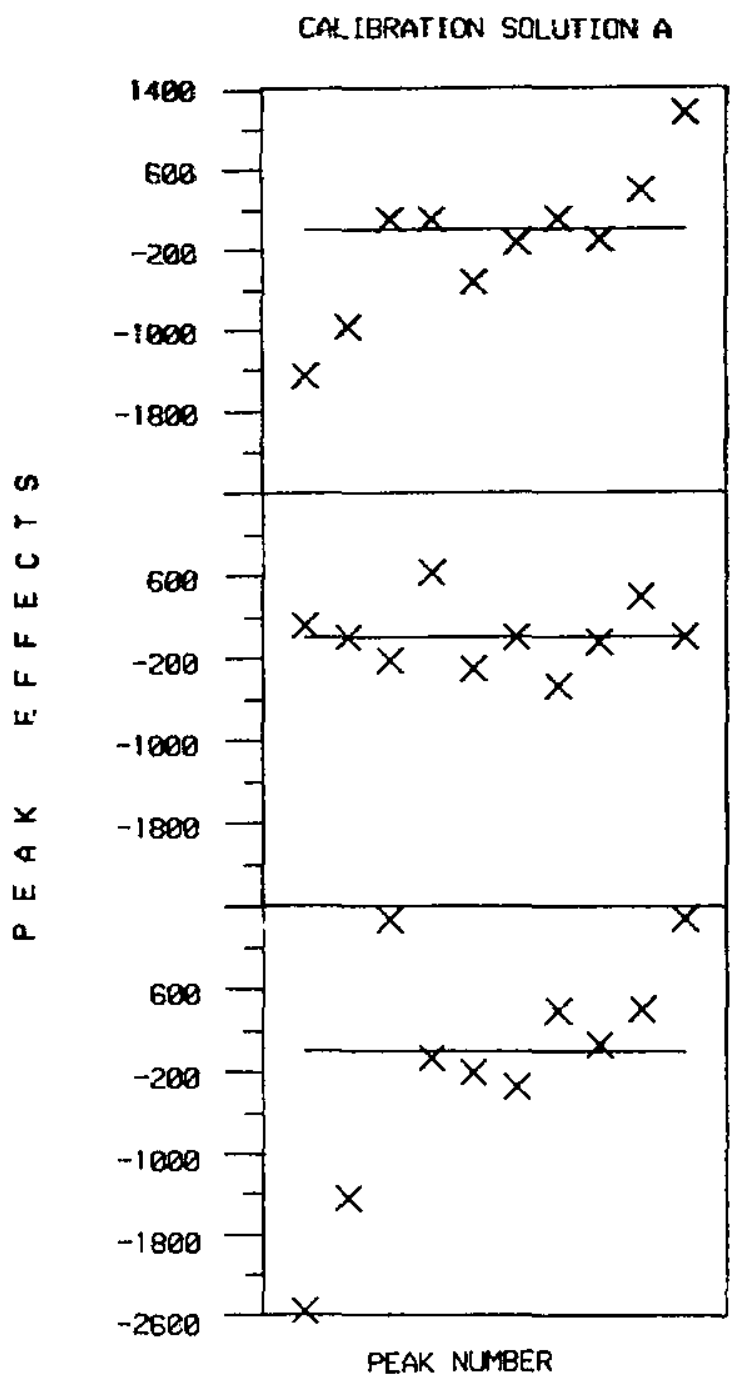

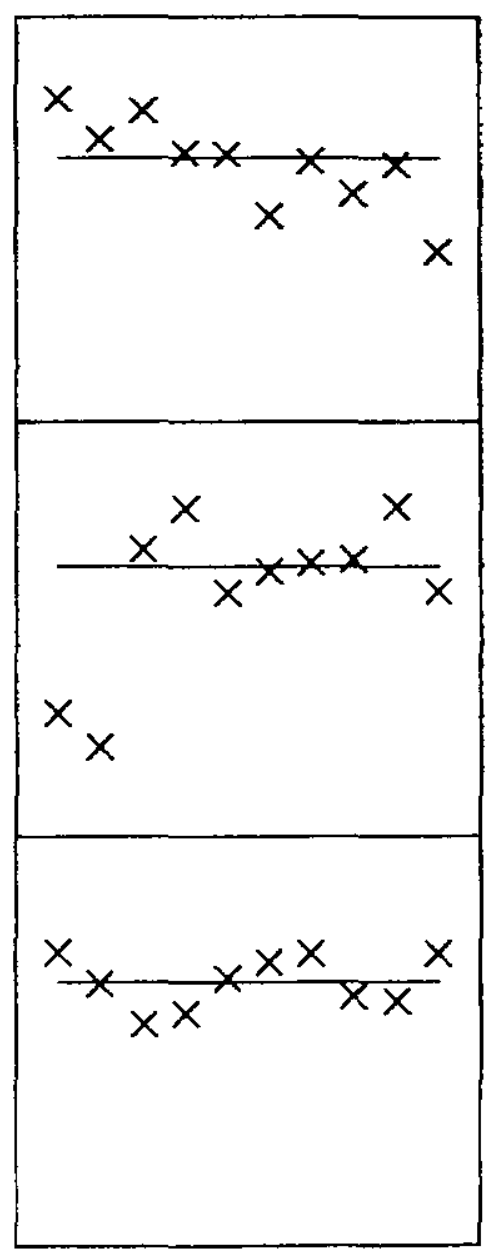

PEAK NUMBER

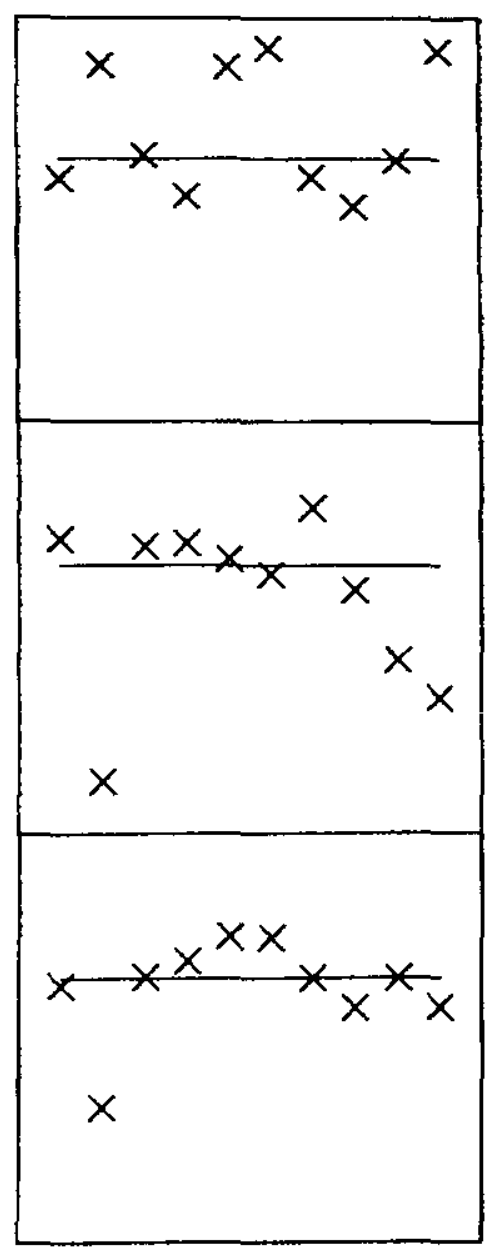

PEAK NUMBER

Figure 4. Peak effects from the median polishes corresponding to the data in figure 3.

We are grateful to Dr. Stephen N. Chesler for his advice on the chemical aspects of this problem and for providing us with the data, to Dr. Walter S. Liggett for his comments on this manuscript and to Dr. Churchill Eisenhart for suggesting the addition of the Appendix.

\section{References}

[1] Tukey, John W. (1977) Exploratory Data Analysis. Reading, MA: Addison-Wesley.

[2] Chesler, S.N., Guenther, F.R., May, W.E., Parris, R.M. (1982) Standard reference materials for accurate analyses of PCBs in oil. ASTM Standardization News, 10, (6); 15-20.

\section{Appendix: Median Polish}

An analysis of a two-way table via median polish may be unfamiliar to many readers, so we present an example using a subset of the PCB data. A typical additive model for a two-way table is

$$
Y_{i j}=\mu+\alpha_{i}+\beta_{j}+\varepsilon_{i j},
$$

where $i=1,2, \ldots$, (number of rows) and $j=1,2, \ldots$, (number of columns). An analysis of variance estimates $\mu$ by $\bar{Y}_{i}$.., $\alpha_{i}$ by $\left(\bar{Y}_{i \bullet}-\bar{Y} ..\right)$ and $\beta_{j}$ by $(\bar{Y} . j-\bar{Y} .$.$) , where dots indicate$ averaging over the missing subscripts. Median polish offers more robust estimates of these parameters as illustrated below. 


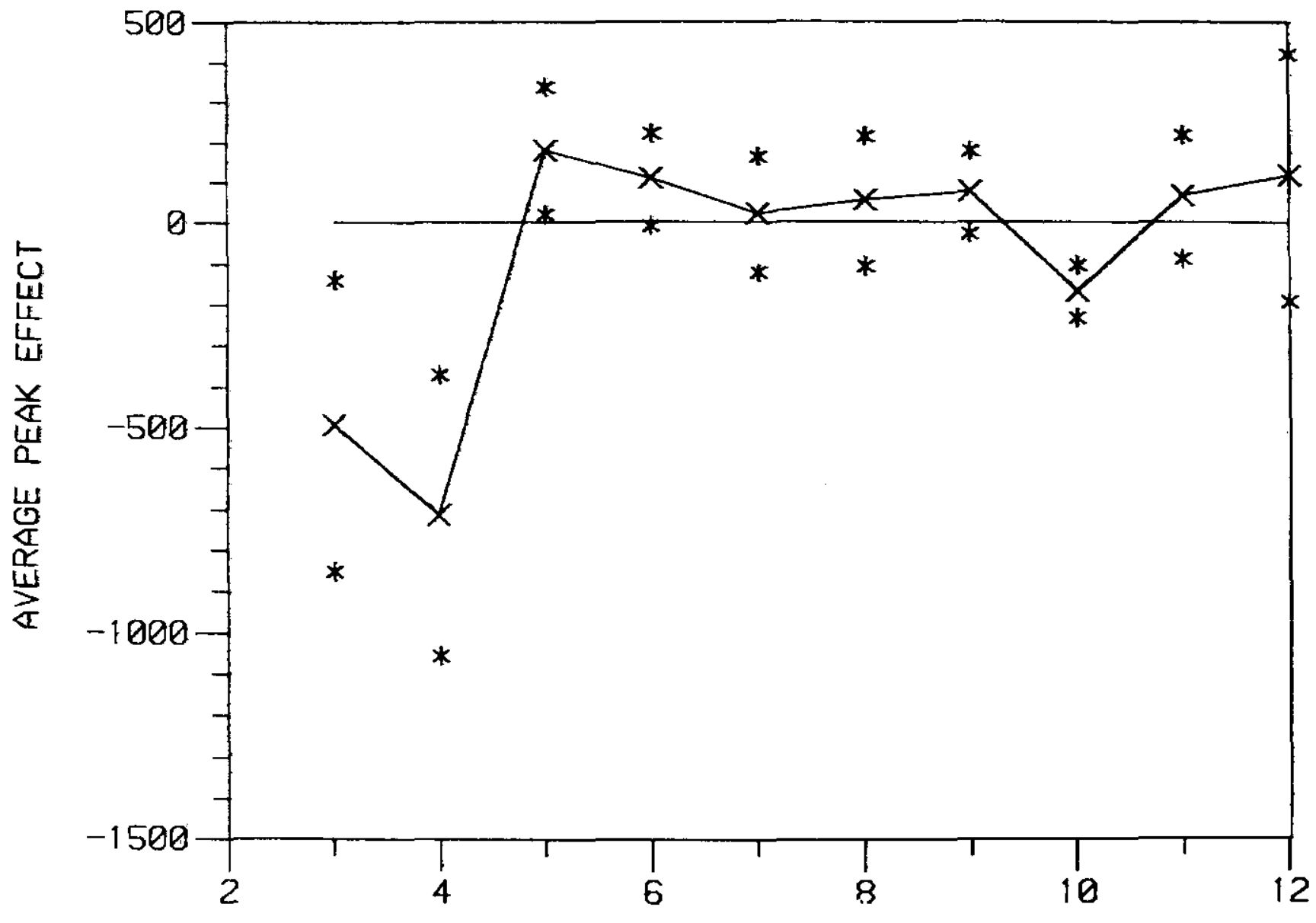

PEAK NUMBER

FIGURE 5. Average peak effect (averaged across all nine replications shown in figure 4). Limits of one standard error of the average shown by *.

Recall from section 4.3 that for each replication $(j=1,2,3)$ of each calibration run $(\alpha=\mathrm{A}, \mathrm{B}, \mathrm{C})$, we have thirty values, $Z_{\alpha j l n}$, corresponding to the three internal standards $(l=1,2,3)$, and 10 Aroclor peaks $(n=3, \ldots, 12)$. These are the thirty values $Y_{i j}$ in the two-way table.

For the data set of Aroclor 1260 in Motor Oil, $j=1$ (first replication) and $\alpha=\mathrm{A}$ (calibration solution), of $\log$ (Aroclor concentration), part of the two-way table is shown below:

\begin{tabular}{|l|ccccc|}
\hline \multicolumn{5}{|c}{} & \multicolumn{5}{c}{ Aroclor Peak } \\
\multicolumn{1}{|c|}{3} & 4 & 5 & 6 & 7 \\
\hline IS 1 & 1.9937 & 1.9985 & 2.0093 & 2.0093 & 2.0030 \\
IS 2 & 1.9827 & 1.9873 & 1.9983 & 1.9983 & 1.9920 \\
IS 3 & 1.9761 & 1.9809 & 1.9917 & 1.9917 & 1.9892 \\
\hline
\end{tabular}

To simplify the arithmetic, let us subtract 2 from each of the numbers and multiply by $10^{4}$ :

\begin{tabular}{|l|rrrrr|}
\hline \multicolumn{1}{|c}{} & \multicolumn{5}{c|}{ Aroclor Peak } \\
\multicolumn{1}{|c|}{3} & 4 & 5 & 6 & \multicolumn{1}{c|}{7} \\
\hline IS 1 & -63 & -15 & 93 & 93 & 30 \\
IS 2 & -173 & -127 & -17 & -17 & -80 \\
IS 3 & -239 & -191 & -83 & -83 & -108 \\
\hline
\end{tabular}

As a first step in estimating the column effects, we write down the median of each column below a single line:

\begin{tabular}{|l|rrrrr|}
\hline \multicolumn{5}{|c}{} & \multicolumn{5}{c|}{ Aroclor Peak } \\
\hline IS 1 & \multicolumn{1}{c}{3} & 4 & 5 & 6 & \multicolumn{1}{c|}{7} \\
IS 2 & -63 & -15 & 93 & 93 & 30 \\
IS 3 & -173 & -127 & -17 & -17 & -80 \\
& -239 & -191 & -83 & -83 & -108 \\
\hline & -173 & -127 & -17 & -17 & -80 \\
\hline
\end{tabular}


We subtract each median from the value in the cell, and put a double line between the table and the medians to indicate that a number has been subtracted:

\begin{tabular}{|l|rrrrr|}
\hline \multicolumn{7}{|c}{} & \multicolumn{5}{c|}{ Aroclor Peak } \\
\multicolumn{2}{|c|}{3} & 4 & 5 & 6 & \multicolumn{1}{c|}{} \\
\hline IS 1 & 110 & 112 & 110 & 110 & 110 \\
IS 2 & 0 & 0 & 0 & 0 & 0 \\
IS 3 & -66 & -64 & -66 & -66 & -28 \\
\hline \hline Column effect & -173 & -127 & -17 & -17 & -80 \\
\hline
\end{tabular}

Next we operate on the rows in the same fashion. First, write down the median of each row (including the row corresponding to the column effects):

\begin{tabular}{|c|c|c|c|c|c|c|}
\hline & & & clor & & & \\
\hline & 3 & 4 & 5 & 6 & 7 & \\
\hline IS 1 & 110 & 112 & 110 & 110 & 110 & 110 \\
\hline IS 2 & 0 & 0 & 0 & 0 & 0 & 0 \\
\hline IS 3 & -66 & -64 & -66 & -66 & -28 & -66 \\
\hline Column effect & -173 & -127 & -17 & -17 & -80 & -80 \\
\hline
\end{tabular}

and then subtract the row medians from the values in the table (again indicated by the double line):

\begin{tabular}{|c|c|c|c|c|c|c|}
\hline & & & clor & & & Row \\
\hline & 3 & 4 & 5 & 6 & 7 & effect \\
\hline IS 1 & 0 & 2 & 0 & 0 & 0 & 110 \\
\hline IS 2 & 0 & 0 & 0 & 0 & 0 & 0 \\
\hline IS 3 & 0 & 2 & 0 & 0 & 38 & -66 \\
\hline Column effect & -93 & -47 & 63 & 63 & 0 & -80 \\
\hline
\end{tabular}

Now we return to columns again: write down the median of each column below the table (single line):

\begin{tabular}{|c|c|c|c|c|c|c|}
\hline & \multicolumn{5}{|c|}{ Aroclor Peak } & \multirow{2}{*}{$\begin{array}{l}\text { Row } \\
\text { effect }\end{array}$} \\
\hline & 3 & 4 & 5 & 6 & 7 & \\
\hline IS I & 0 & 2 & 0 & 0 & 0 & 110 \\
\hline IS 2 & 0 & $\mathbf{0}$ & $\mathbf{0}$ & 0 & $\mathbf{0}$ & 0 \\
\hline IS 3 & 0 & 2 & 0 & 0 & 38 & -66 \\
\hline \multirow[t]{2}{*}{ Column effect } & -93 & -47 & 63 & 63 & 0 & -80 \\
\hline & 0 & 2 & $\mathbf{0}$ & 0 & $\mathbf{0}$ & $\mathbf{0}$ \\
\hline
\end{tabular}

Subtract these medians from the values in the corresponding column, and add the two sets of column effects together:

\begin{tabular}{|c|c|c|c|c|c|c|}
\hline & \multicolumn{5}{|c|}{ Aroclor Peak } & \multirow{2}{*}{$\begin{array}{l}\text { Row } \\
\text { effect }\end{array}$} \\
\hline & 3 & 4 & 5 & 6 & 7 & \\
\hline IS 1 & 0 & 0 & 0 & 0 & 0 & 110 \\
\hline IS 2 & 0 & -2 & 0 & 0 & $\mathbf{0}$ & o \\
\hline IS 3 & 0 & $\mathbf{0}$ & 0 & 0 & 38 & -66 \\
\hline Column effect & -93 & -45 & 63 & 63 & $\mathbf{0}$ & -80 \\
\hline
\end{tabular}

Now we return to the rows, the medians of which are easily seen to be all 0's. So there is no further "polishing" of this table. Returning to the original scale, the row effects $\hat{\alpha}_{i}$ (corresponding to internal standards) are $(0.0110$, $0.0000,-0.0066)$, the column effects $\hat{\beta}_{j}$ (corresponding to Aroclor peaks) are (-.0093, -.0045, .0063, .0063, .0000), and the overall typical value $(\hat{\mu})$ is 1.9920 .

Notice that:

- Median polish essentially "converges," in that, beyond a certain point, the table changes little;

- At each stage, the values in the table ("residuals") are further polished, so that, at any stage of the process, row effect + column effect + overall typical value + tabled entry $=$ original data value;

- Whereas the usual analysis of variance summarizes rows and columns by means, median polish summarizes by medians;

- A plot of the peak effects versus peak number would reveal that the effect from peak 3 is considerably lower than the others; and

- An extreme value in the table is flagged by having an unusually large or small residual in the cell of the final table (e.g., IS 3 and Aroclor Peak 7). Most of the entries in the final tableau, however, are nearly zero.

Three- and four-way median polish are also possible; see [1] (pp. 452ff) for an example. 